\title{
Second threshold condition in the case of $Q$ switching by self-focusing
}

\author{
M. C. Marconi, O. E. Martinez, and F. P. Diodati \\ Ceilap, Citefa-Conicet, Zufriategui y Varela, 1603-Villa Martelli, Argentina
}

Received March 7, 1986; accepted September 9, 1986

\begin{abstract}
In a recent paper [Opt. Lett. 10, 402 (1985)] we presented a novel passive technique for obtaining giant laser-pulse emission. In this paper we present a more sophisticated model based on Kirchhoff-Fresnel diffraction theory that confirms the aberrationless Gaussian model used before. Also, a simple criterion for short-pulse generation in the $Q$ switch by the self-focusing technique is derived. A set of equations is obtained that permits the evaluation of the critical parameters that govern the laser dynamics. The second threshold condition is derived, and some typical examples are pointed out.
\end{abstract}

In a recent paper $^{1}$ we presented a novel passive $Q$-switching technique. This new technique makes use of the self-focusing effect in a liquid with a high nonlinear refractive index placed in contact with one of the cavity mirrors of an unstable resonator. At high intensities the self-focusing reduces the diffraction losses by changing the effective power of the mirror, creating a stable-resonator configuration and causing $Q$ switching to occur. In this analysis, we use the Gaussian mode approximation to describe the $Q$-switching technique. With this formalism, the round-trip matrix and the geometric approximation can be used in order to obtain the loss coefficient $L$. This geometrical formalism provides a limited description of the system. More-detailed knowledge of the system evolution must be obtained by solving the Kirchhoff-Fresnel integral. 2,3 We will consider the propagating wave that is reflected back and forth in the cavity equivalent to the transmission of this wave through limiting apertures defined by the laser mirrors. This is the usual approximation in the diffraction analysis of resonant laser cavities. Figure 1 shows the passive cavity that we use and the equivalent scheme used for the analysis. From a given initial intensity profile in the focusing cell $u(r)$, it is possible to obtain the intensity distribution after one round trip $u^{\prime}(r)$. These two spatial profiles are related by the propagator of the cavity ${ }^{4}$

$$
\begin{aligned}
u^{\prime}\left(r_{2}\right)= & \frac{2 \pi i \xi^{2}}{\lambda\left(2 \xi-F_{2}\right)} \psi\left[r_{2}, \xi-\frac{\xi^{2}}{\left(2 \xi-F_{2}\right)}\right] \\
& \times \int_{E 1} u\left(r_{1}\right) \psi\left[r_{1}, \xi-\frac{\xi^{2}}{\left(2 \xi-F_{2}\right)}\right] \\
& \times \exp \left[i \phi_{c}\left(r_{1}\right)\right] J_{0}\left[\frac{2 \pi}{\lambda} \frac{\xi^{2}}{\left(2 \xi-F_{2}\right)} r_{2} r_{1}\right] r_{1} \mathrm{~d} r_{1},
\end{aligned}
$$

where $\xi$ is the inverse of the cavity length $L_{c}, F_{i}$ is the inverse of the mirror focal length, $\phi_{c}(r)$ is the phase shift introduced by the focusing cell, $\psi(r, A)=\exp \left[-(i \pi / \lambda) A r^{2}\right]$, and $\psi^{*}(r, A)$ is the complex conjugate of $\psi(r, A)$. The integral must be calculated over the limiting aperture of the mirror $E_{1}$ with curvature radius $R_{1}$. The eigenfunctions of this propagator will define the stable oscillating modes, and the respective eigenvalues will be the round-trip magnification of the cavi- ty. The stable-mode profile will be a function of the geometrical cavity configuration, and this configuration depends on the focusing characteristics of the cell. As the phase shift introduced by the focusing cell depends on the cavity intensity, the stable modes will also be intensity dependent.

We made numerical calculations to compute such stable modes. The numerical simulations compute the spatial profile in each round trip by using Eq. (1). The profile obtained in each round trip is normalized at a given peak intensity and is compared with the previous normalized profile. This sequence continues until the two profiles coincide. For different normalizing peak intensities, different stable modes are obtained. The same stable intensity distribution is obtained when starting from different initial profiles.

For the initial profile we used a plane-front wave with a constant amplitude and a plane-front wave with a Gaussian amplitude distribution. The same stable mode is obtained with both initial profiles, but in the latter case the solution is reached faster.

In the aberrationless formalism used in Ref. 1, the spot size of the stable lower-order mode is fixed by the cavity geometry. The spot size in the mirror $\mathrm{E}_{1}$ is thus given by

$$
a_{1}^{4}=\left(\frac{\lambda R_{1}}{\pi}\right)^{2} \frac{R_{2}-L_{c}}{R_{1}-L_{c}} \frac{L_{c}}{R_{1}+R_{2}-L_{c}} .
$$

In Eq. (2) $R_{1}$ can be replaced by its expression as a function of the field intensity. In this way, a quadratic equation for $a^{2}$ is obtained. Solving this equation, we obtain the curve plotted in the Fig. 2. This curve represents the spot size in the mirror $\mathrm{E}_{1}$ as a function of the circulating intensity in the cavity, if we assume that the focusing cell acts as a spherical thin lens. In our simulations we use a Gaussian profile with a spot size given by Eq. (2) as the initial intensity distribution. For small intensity values, a broad profile characteristic of unstable resonators is obtained. This is reasonable because, at lower intensities, the influence of the focusing cell must be negligible. At higher intensities, the profile approaches a Gaussian distribution except in the tails at lower intensities. 


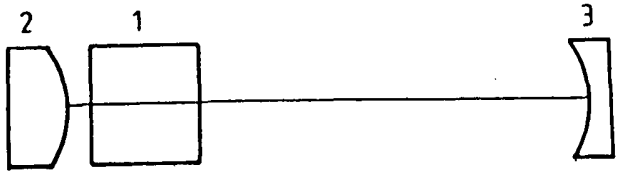

$u(r)$

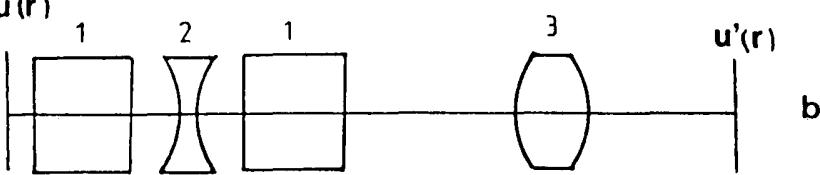

Fig. 1. a, Passive-cavity scheme: 1, focusing cell; 2, divergent mirror; 3, convergent mirror. b, Equivalent optical system. The initial and the final profiles are shown.

An important result may be obtained from this diffraction formalism. For the stable mode, it is possible to obtain the round-trip loss coefficient as the ratio between the peak intensities of the initial and final profiles. In Fig. 3 the round-trip loss coefficient as a function of the intensity (in watts per square centimeter) for the Gaussian-model approach (dashed lines) and the diffraction formalism (circles) is plotted. For intensities corresponding to a stable-cavity configuration, reasonable agreement between the two models is obtained, both for the loss and for the phase shift. In the high-energy-loss branch (negative-branch resonators) the apparent difference between the two models can be explained by taking into account that in the first model (Gaussian approach) the spot size is considered a constant. By changing this value within the values shown in Fig. 2, a better fit can be obtained. The validity of the numerical results obtained for the intensity evolution with the simplified model of Ref. 1 is thus justified. Moreover, the numerical predictions are confirmed by the experimental results.

Another important subject that we discuss in this paper is the conditions that must be fulfilled in order to achieve $Q$ switching. As was shown by New and $\mathrm{O}^{\prime} \mathrm{Hare},{ }^{6}$ a second threshold condition exists in short-pulse generation by passive techniques. ${ }^{7}$ In order for single-pulse emission to be obtained by $Q$ switching the cavity, the initial increase in the net gain coefficient resulting from loss reduction must be larger than the initial decrease resulting from amplifier depletion by stimulated emission. Hence the giant-pulse condition can be expressed as

$$
\frac{\mathrm{d} L}{\mathrm{~d} t}<\frac{\mathrm{d} A}{\mathrm{~d} t}
$$

where $L$ is the diffraction loss coefficient. In the self-focusing method that we consider here, the diffraction-loss coefficient $L$ is intensity dependent through the cavity parameter g. The loss coefficient per round trip $L$ can be obtained from the cavity configuration that is intensity dependent ${ }^{1}$ :

$$
L=2 \ln \left[g \pm\left(g^{2}-1\right)^{1 / 2}\right],
$$

where + and - correspond to positive- and negative-branch resonators, respectively, and $g$ is the cavity parameter., 8,9 The parameter $g$ determines the stability characteristics of the cavity, which will be stable for $0<\mathrm{g}<1$.

The intensity dependence of $g$ can be obtained from the cavity configuration, and this configuration can be described by using the matrix formalism. ${ }^{10,11}$ This simple formalism is an aberrationless approach that is in good agreement with more-realistic models and with experimental results, as was pointed out before. In this case we obtain

$$
g=g_{0}-\beta h \nu \phi
$$

where $g_{0}$ is the initial value of parameter $g$ defined by the initial cavity configuration, $\phi$ is the photon-flux density in the cavity, and $\beta$ is a parameter that describes the nonlinear characteristics of the focusing medium. In the definition of the parameter $\beta$, the nonlinear characteristics of the focusing medium and the cavity configuration are both included. As was defined in Ref. 1,

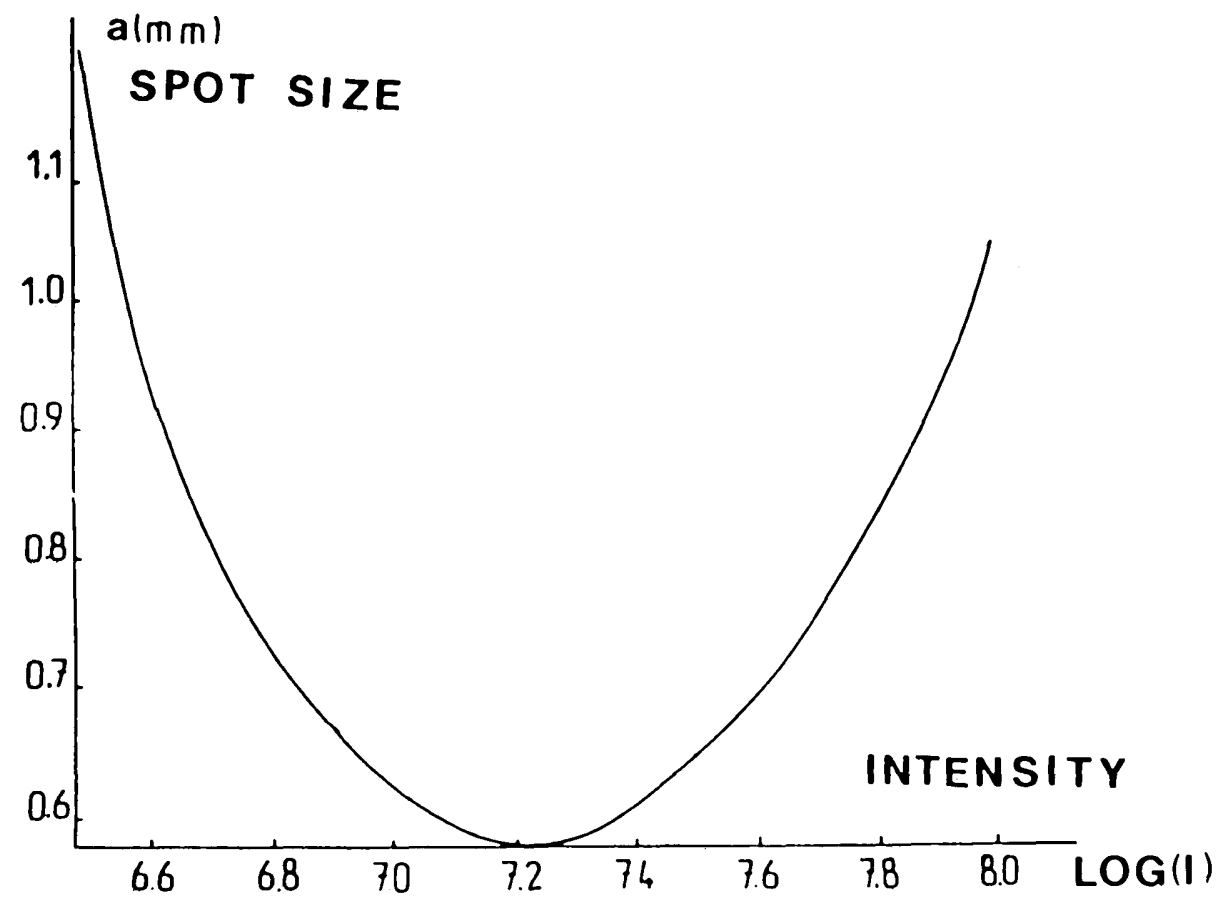

Fig. 2. Spot size versus the circulating cavity intensity (watts per square centimeter) in the Gaussian mode approach. 


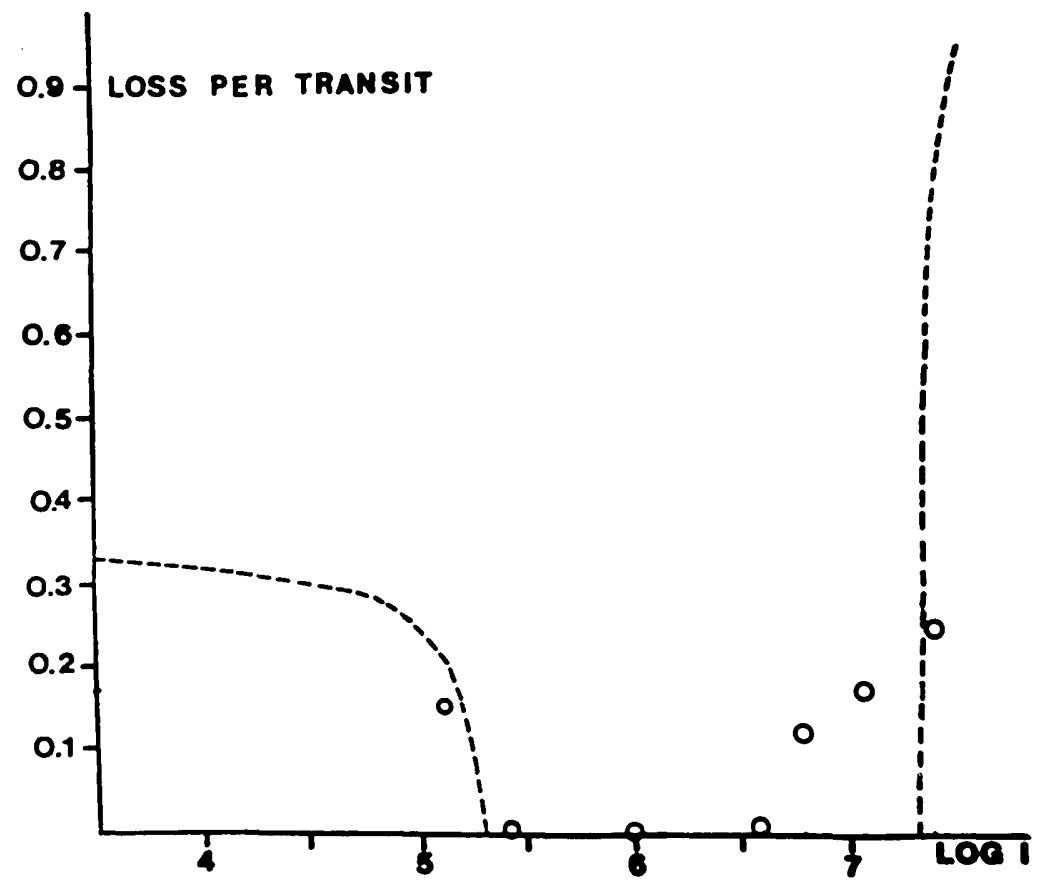

Fig. 3. Cavity loss versus the circulating cavity intensity (watts per square centimeter). Dashed lines, Gaussian mode approach; circles, loss coefficients obtained with the diffraction formalism.

$$
\beta=2 L_{c} \alpha \gamma\left(1-L_{c} / R_{i}\right)
$$

In this definition, $\alpha=2 d / a^{2}$, where $d$ is the focusing-cell length and the parameter $a$ is the spot size in the cell. $L_{c}$ is the cavity length, and $\gamma=4.19 \times 10^{-3} n_{2} / n_{0}\left(\mathrm{~cm}^{2} / \mathrm{W}\right)$ (Ref. 12) taking into account the nonlinear characteristics of the cell. In this expression, $R_{i}$ is the curvature radius of the mirror that is not in contact with the focusing cell. When this dependence is taken into account, the diffraction-loss coefficient as a function of time can be expressed as

$$
\frac{\mathrm{d} L}{\mathrm{~d} t}=\frac{\partial L}{\partial g} \frac{\partial g}{\partial \phi} \frac{\partial \phi}{\partial t}
$$

Replacing the partial derivatives in Eq. (7), we obtain

$$
\frac{\mathrm{d} L}{\mathrm{~d} t}=\frac{2}{\left(g^{2}-1\right)^{1 / 2}} \beta h \nu(A-L-\Gamma) \phi / T_{\text {cav }},
$$

where $\Gamma$ is the linear loss coefficient (external coupling, scattering, absorption, etc.) and $T_{\text {cav }}$ is the round-trip time. By substituting Eq. (8) into expression (3) the $Q$-switching condition is given by

$$
\left[\sigma A-\frac{2 \beta h \nu(A-L-\Gamma)}{\left(g^{2}-1\right)^{1 / 2} T_{\text {cav }}}\right] \phi<P,
$$

where $\sigma$ is the amplifier cross section and $P$ is the pumping rate. The first term of inequality (9) represents the amplifier depletion by stimulated emission, and the second term is the diffraction-loss reduction. In order to obtain a more general expression of inequality (9), we use dimensionless variables. We define the dimensionless photon flux $\mu$ and the dimensionless time $k$ as

$$
\begin{aligned}
& \mu=\beta h \nu \phi /\left|g_{0}-1\right|, \\
& k=t / T_{\text {cav }} .
\end{aligned}
$$

The rate equations for the photon flux, the amplification coefficient, and the cavity parameter $g$ expressed with these dimensionless variables are

$$
\begin{aligned}
\frac{\mathrm{d} \mu}{\mathrm{d} k} & =(A-L-\Gamma) \mu, \\
\frac{\mathrm{d} A}{\mathrm{~d} k} & =\left|g_{0}-1\right| \frac{\mu \sigma A T_{\text {cav }}}{\beta h \nu}+P, \\
g & =g_{0}-\left|g_{0}-1\right| \mu .
\end{aligned}
$$

The laser dynamics in the $Q$-switching process can be analyzed in three distinct stages: (1) The linear amplification stage begins when the system crosses the laser threshold and continues until the time when the reduction in the cavity losses by the self-focusing begins to be significant; (2) the loss saturation stage starts when the intensity reaches a value for which the change in the diffraction losses are significant; (3) the gain saturation stage occurs when, because of the high intensity in the cavity, the amplifier medium is depleted and the laser action ends.

The linear amplification stage concludes when the reduction of the losses is significant and the net gain grows quickly, i.e., in a few round trips. This moment defines a time $k_{\text {sat }}$ that depends on the cavity configuration and the nonlinear characteristics of the focusing cell. We obtain an expression for $k_{\text {sat }}$ and evaluate the different parameters included in the second threshold equation at this stage. The slope of the loss coefficient $L$ is a monotonically decreasing function of $\mu$ until the cavity becomes stable, and that ensures that the second threshold condition [expression (3)] will be fulfilled at higher intensities if it is fulfilled at $k_{\text {sat. }}$. In the linear amplification stage, we can consider

$$
\begin{aligned}
& A=A_{0}+P k, \\
& L=L_{0}-b \mu .
\end{aligned}
$$


If we substitute Eqs. (12a) and (12b) into Eq. (11a) an equation that describes the photon flux evolution in the first stage is obtained:

$$
\frac{\mathrm{d} \mu}{\mathrm{d} k}=(P k+b \mu) \mu,
$$

where we used the threshold condition $A_{0}-L_{0}-\Gamma=0$. Equation (13) can be expressed as

$$
\frac{\mathrm{d} x}{\mathrm{~d} k}=(P k+x) x
$$

where $x=b \mu$.

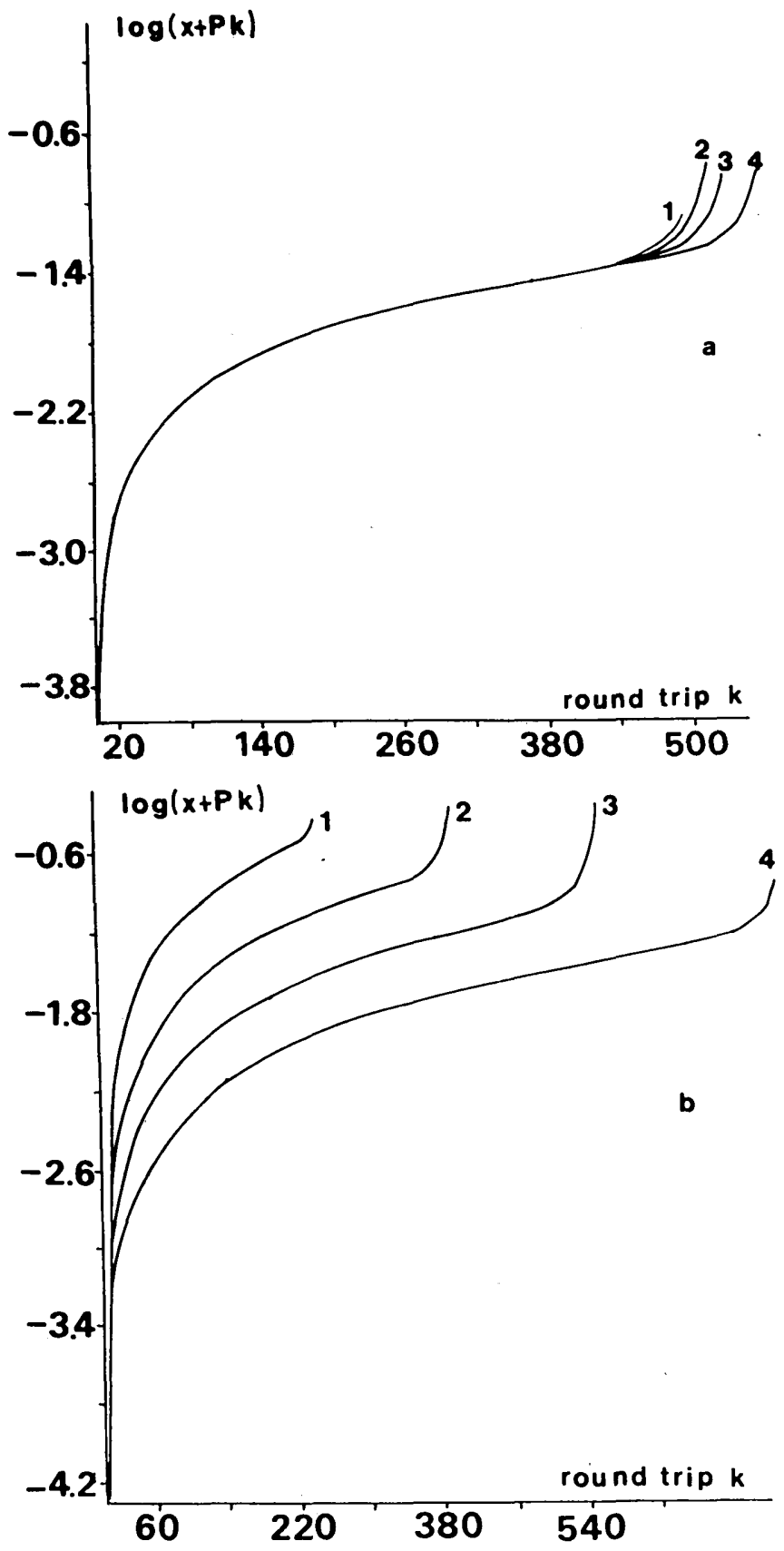

Fig. 4. Net gain $(x+P k)$ versus the round-trip number $k$. a, For different values of the initial photon density $x_{0}: 1, x_{0}=12 \times 10^{-8} ; 2$, $x_{0}=8 \times 10^{-8} ; 3, x_{0}=4 \times 10^{-8} ; 3, x_{0}=10^{-8}$. b, For different values of the pump rate $P: 1, P=5 \times 10^{-4} ; 2, P=2 \times 10^{-4} ; 3, P=10^{-4} ; 4, P=$ $5 \times 10^{-5}$
In Fig. 4 the numerical results of Eq. (14) for the net gain $(P k+x)$ are plotted, changing the initial photon flux $x_{0}$ and the pumping rate $P$. Figure 4 a shows the solution for different values of the initial photon flux $x_{0}$. In this case, the $k_{\text {sat }}$ (when the net gain grows during a few round trips) has nearly the same value for the different values of $x_{0}$ considered. Figure $4 \mathrm{~b}$ shows the results for different pumping rates $P$ and shows the strong dependence of $k_{\text {sat }}$ on the pumping rate $P$. The pumping rate $P$ will be the dominant parameter in Eq. (13).

Substituting $\delta=1 / x$ into Eq. (14), we obtain

$$
\frac{\mathrm{d} \delta}{\mathrm{d} k}=-P k \delta-1 \text {. }
$$

Solving Eq. (15), we obtain

$$
\delta=\exp \left(-\frac{P k^{2}}{2}\right)\left[-\int_{0}^{k} \exp \left(\frac{P k^{\prime}}{2}\right) \mathrm{d} k^{\prime}+\delta_{0}\right] .
$$

Equation (16) is the solution of the Eq. (13), and it has two terms. The first one $(P k)$ is the net gain increase by the pumping rate. The second one $(b \mu)$ is the gain increase by the diffraction-loss reduction. At low intensities, the first term is the dominant one, and this regime defines the linear amplification stage. Because of the high nonlinear effect in the focusing cell, the second term begins to be important at high intensities. We can assume that the end of the linear amplification stage will be defined by the moment when these two terms have the same value. With the condition that $P k=b \mu$, Eq. (16) gives

$$
-(2 P)^{1 / 2} \mathbf{D}\left(\sqrt{\frac{P}{2}}_{k}\right) k+P \delta_{0} \exp \left(-P k^{2} / 2\right) k=1,
$$

where $\mathbf{D}(x)$ is a Dawson integral, defined as ${ }^{13}$

$$
\mathbf{D}(x)=\exp \left(-x^{2}\right) \int_{0}^{x} e^{t^{2}} \mathrm{~d} t
$$

Using the asymptotic value for $\mathbf{D}(x)=0.5197 / x$ (for $x>4.5$ ), we obtain

$$
-1.04+P k \delta_{0} \exp \left(-P k^{2} / 2\right)=1 .
$$

Finally, from Eq. (19) it is possible to derive an expression for $k_{\text {sat }}$ :

$$
k_{\text {sat }}=\left[\frac{2}{P} \ln \left(\frac{P k \delta_{0}}{2.04}\right)\right]^{1 / 2},
$$

which can be solved by iterations. The dominant parameter in this expression, as was mentioned before, is the pumping rate $P$. Figure 5 shows the solution of Eq. (20) for different values of the initial photon flux $x_{0}$. The $k_{\text {sat }}$ values obtained from Fig. $4 \mathrm{~b}$ are indicated by circles. The agreement between the asymptotic approximation [Eq. (20)] and the numerical simulations is within $5 \%$.

The last parameter that must be evaluated in order to obtain a complete set of equations is the parameter $b$ in Eq. (12b). If we evaluate $\mathrm{d} L / \mathrm{d} \mu$, for the first stage with $\mu \ll 1$ we obtain

$$
\frac{\mathrm{d} L}{\mathrm{~d} \mu}=\frac{\partial L}{\partial g} \frac{\partial g}{\partial \mu}=-\left[\frac{4\left(g_{0}-1\right)}{g_{0}+1}\right]^{1 / 2}=-b .
$$

Now it is possible to evaluate the critical parameters that govern the laser dynamics in the end of the linear amplifica- 


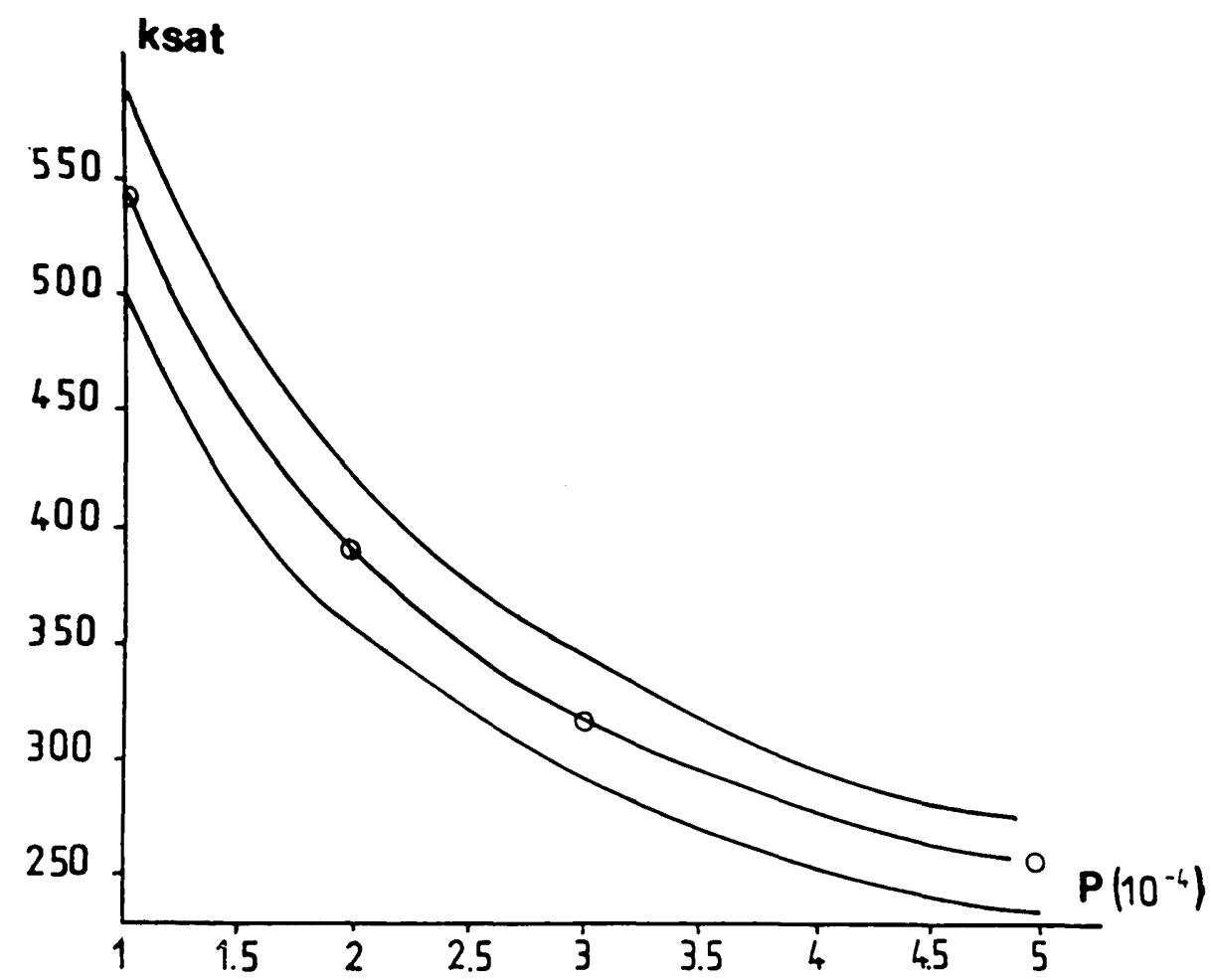

Fig. 5. Solution of Eq. (20). The curves represent the saturation time $k_{\text {sat }}$ as a function of the pumping rate $P$ for different values of the initial photon flux $x_{0}$ : top curve, $x_{0}=10^{-9}$; middle curve, $x_{0}=10^{-8}$; bottom curve, $x_{0}=10^{-7}$. The circles are the $k_{\text {sat }}$ values obtained from Fig. $4 \mathrm{~b}$.

tion stage by using Eqs. (20) and (21) and the following three equations that can be obtained from Eqs. (4), (12a), and (16):

$$
\begin{aligned}
L & =2 \ln \left[g_{0}+\left|g_{0}-1\right| \mu \pm\left(g_{0}^{2}-1\right)\right], \\
A & =A_{0}+P k, \\
\frac{1}{b \mu} & =\frac{\exp \left(-P k^{2} / 2\right)}{b \mu_{0}}-\sqrt{\frac{2}{P}} \mathbf{D}\left(\sqrt{\frac{P}{2}} k\right) .
\end{aligned}
$$

In the Eq. (22a) the + or - must be used if an initial positive- or negative-branch configuration, respectively, is considered.

Equations (22) allow us to evaluate the second threshold condition, which, expressed as a function of the dimensionless parameter $\mu$, is

$$
\frac{\sigma T_{\mathrm{cav}} A\left|g_{0}-1\right| \mu}{\beta h \nu P}-\frac{2(A-L-\Gamma) \mu}{\left(\frac{g_{0}+1}{g_{0}-1}\right)^{1 / 2} P}<1 .
$$

Expression (23) must be fulfilled in order to obtain the $Q$ switching of the cavity. The fulfillment of this condition will depend on the laser-amplifier characteristics and on the cavity configurations. The initial cavity configuration defines the $g_{0}$ value and the initial loss per round trip. If a $\mathrm{Nd}$ :glass system is used, the maximum value for $L$ will be about $30 \%$ per round trip. As an example, we discuss the design criteria for a cavity length of about $1 \mathrm{~m}$ with two totally reflecting mirrors similar to that described in Ref. 1. The maximum initial loss admissible sets the initial value of the cavity parameter $g_{0}=1.016$. This value can be obtained with two configurations: (a) with one divergent and one convergent mirror (curvature radius $R_{1}=2 \mathrm{~m}, R_{2}=-1 \mathrm{~m}$, and a cavity length $L_{c}=0.985 \mathrm{~m}$ ) for the positive-branch configuration; (b) with two convergent mirrors (curvature radius $R_{1}=1 \mathrm{~m}, R_{2}=2 \mathrm{~m}$, and a cavity length $L_{c}=1.016 \mathrm{~m}$ ) for the negative-branch configuration.

In the positive-branch configuration, the focusing cell can be placed in contact with the convergent mirror $\mathrm{E}_{1}$ or with the divergent mirror $\mathrm{E}_{2}$. In Fig. 6 , the g parameter as a function of the equivalent focal length of the focusing cell $z_{f}$ is plotted. Only the stable zone $(0<g<1)$ for the two possibilities mentioned before is plotted. With the cell touching the convergent mirror $\mathrm{E}_{1}$, the system rapidly reaches a unstable configuration. This would drastically reduce the energy obtainable. In case (b) (negative-branch resonator), the focusing cell must be placed in contact with mirror $\mathrm{E}_{2}$ (radius of curvature $2 \mathrm{~m}$ ). This is a unique choice in this case because the cell in contact with the mirror $\mathrm{E}_{1}$ will give a cavity that becomes more unstable as the intensity grows. With the negative-branch configuration, the cavity parameter $g$ has a slight dependence on the field intensity. If we evaluate the function $\mathrm{dg} / \mathrm{d} \mu$, it can be demonstrated that in case (b) this variation rate is about 100 times smaller than in positive-branch configuration. For this reason [see Eq. (7)] the second threshold condition cannot be fulfilled.

The stability condition, as we mentioned before, is

$$
0 \leqslant g=g_{1} g_{2} \leqslant 1,
$$

where $g_{i}=1-L_{\mathrm{c}} / R_{i}$.

The stable boundary zones can be plotted in the $g_{1}-g_{2}$ plane. In Fig. 7, the $g_{1}-g_{2}$ plane with the cavity evolution for the three cases discussed is plotted. The two initial configurations are also shown: point $\mathrm{A}$ for the positive-branch resonator and point $B$ for the negative-branch resonator. In case (b), the cavity evolution is along a straight line with a small $g_{1}$, and thus the $g$ parameter has a low intensity depen- 


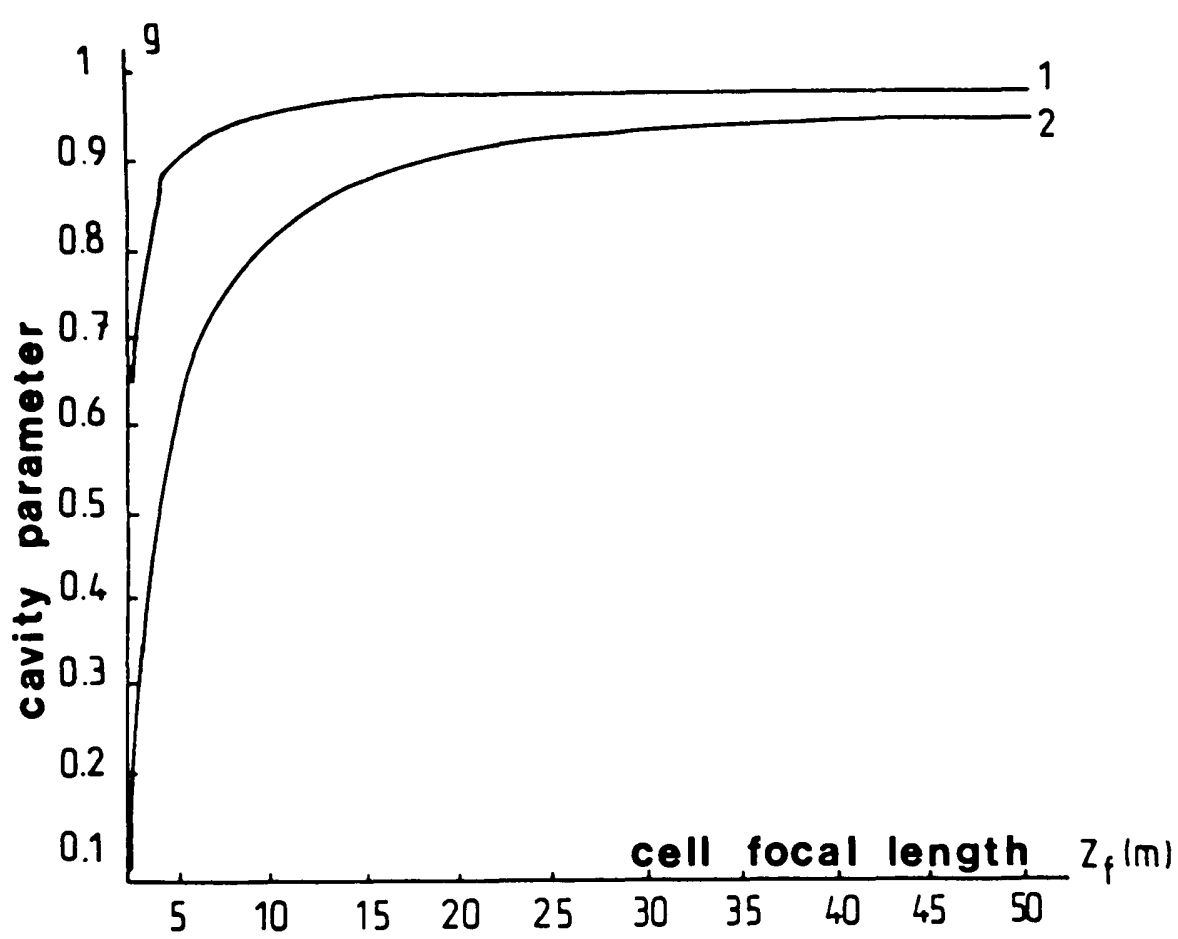

Fig. 6. Cavity parameter $g$ as a function of the focal length $z_{f}$ of the focusing cell: 1 , with the cell in contact with the divergent mirror $E_{2} ; 2$, with the cell in contact with the convergent mirror $E_{1}$.

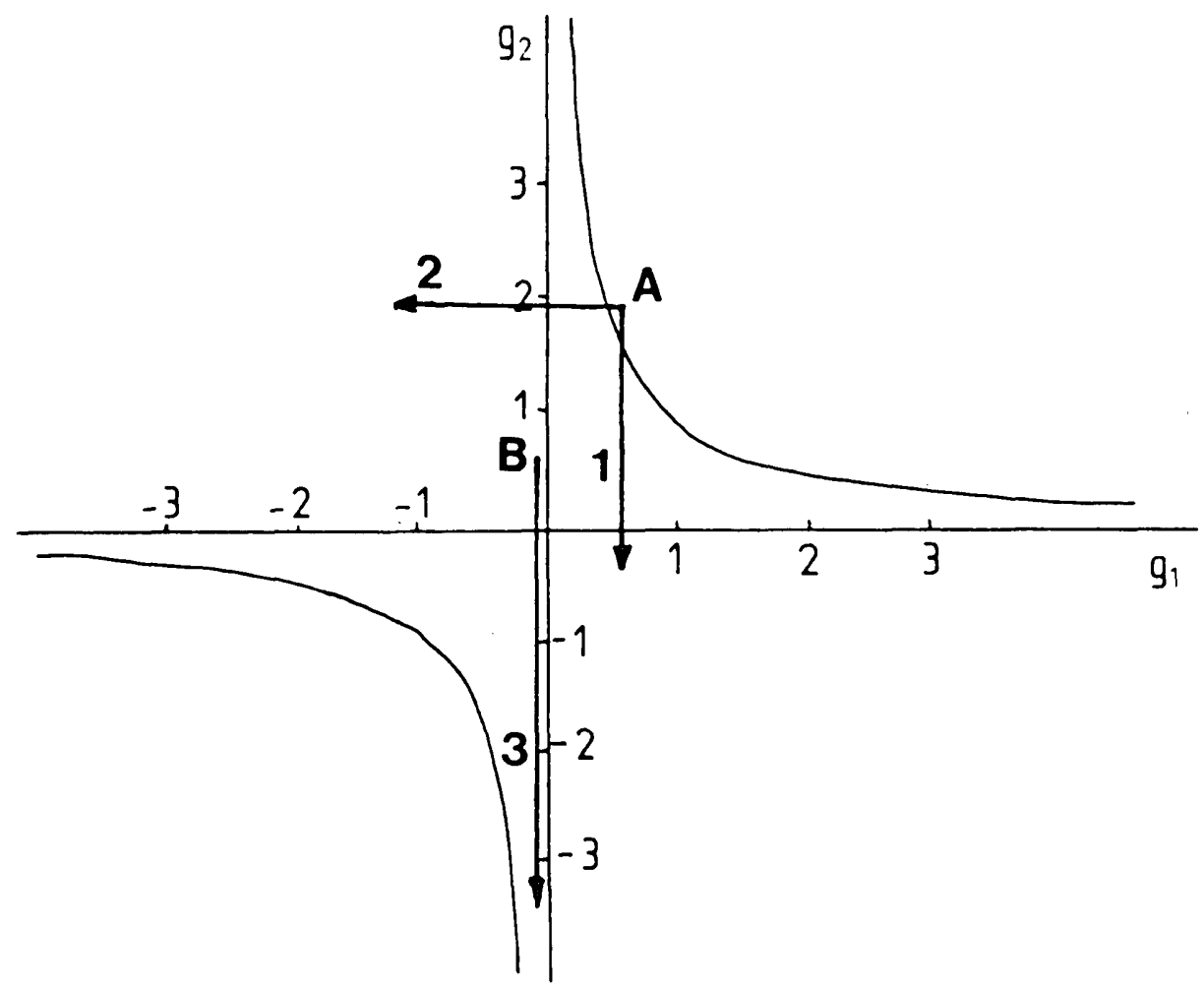

Fig. 7. Stable frontier zones in the $g_{1}-g_{2}$ plane. The initial configurations are plotted with the cavity evolution for the different cases discussed. A, Initial configuration for the positive-branch resonator. 1 is the cavity evolution obtained with the focusing cell in contact with the divergent mirror and 2 is that when the cell is in contact with the convergent mirror. B, Initial configuration for the negative-branch resonator. 3 is the cavity evolution in this case.

dence. As can be concluded, the best choice for the cavity design is a positive-branch resonator with the focusing cell in contact with the divergent mirror.

Finally, we present some numerical examples using the design equations in order to evaluate the $Q$-switching condi- tion [expression (23)] for a Nd:glass system, with both configurations (positive- and negative-branch) and for a $\mathrm{Nd}$ :YAG amplifier.

For a Nd:glass amplifier we consider $A_{0}=1.3, \sigma=0.4 \times$ $10^{-19} \mathrm{~cm}^{2}, P=10^{-4}, \beta=10^{-8} \mathrm{~cm}^{2} / \mathrm{W}, \phi_{0}=10^{18} \mathrm{~cm}^{-2} \mathrm{sec}^{-1}, g_{0}$ 
$=1.016$, and $T_{\text {cav }}=6$ nsec. The $\beta$ value was evaluated using phenyl salicylate ${ }^{14}$ as the nonlinear medium. With these initial values, using Eqs. (21), (10a), and (20), we obtain $b=$ $0.178, \mu_{0}=1.156 \times 10^{-7}$, and $k_{\text {sat }}=530$. Substituting these values into Eqs. (22), we obtain the laser parameters at the end of the linear amplification stage: $A=1.353, \mu=0.273$, and $L=0.349$. Finally, substituting these values into expression (23), we obtain

$$
7-145<1 \text {. }
$$

In this case $\mathrm{d} L / \mathrm{d} t$ is 20 times larger than $\mathrm{d} A / \mathrm{d} t$, and in this way the second threshold condition is fulfilled. If we consider a positive-branch resonator with the focusing cell in contact with the convergent mirror, we must change the value of the parameter $\beta$. In this case, $\beta$ is four times larger than in the former case. With these values of the initial parameters we obtain $\mu_{0}=4.612 \times 10^{-7}$ and the same values for the other parameters. Substituting these values into expression (23), we obtain

$$
4-310<1
$$

In this case also the second threshold condition is fulfilled. The last example that we will consider with a Nd:glass amplifier is the negative-branch configuration. In this case, we obtain $g_{1}=-0.016, g_{2}=0.492$, and then $g_{0}=-1.016$. With this value for the initial cavity parameter, we obtain $b=$ $22.45, \mu_{0}=9.17 \times 10^{-10}$, and $k_{\text {sat }}=530$. When these values are substituted into Eqs. (22), the laser parameters at the end of the linear amplification stage are $A=1.353, \mu=0.002$, and $L=0.301$. If we substitute them into expression (23) we obtain

$$
214-134>1 \text {. }
$$

In this case, as we pointed out before, the $Q$-switching condition is not achieved.

If a Nd:YAG laser system is used, the emission cross section is about 20 times larger than for the $\mathrm{Nd}$ :glass laser. Moreover, as the net gain is about 20 times higher, the initial cavity configuration must be strongly unstable, and the initial cavity parameter $g_{0}$ can be as large as 15 instead of 1.016 . This adds a factor of 800 in the term $\left|g_{0}-1\right|$. In the YAG system, the gain decrease by stimulated emission [the first term in expression (23)] will always be larger than the loss decrement [the second term in expression (23)]. The loss saturation stage cannot be achieved, and a giant pulse is not emitted.

The parameter that defines the characteristics of the amplifier in the second threshold condition is the amplifier cross section $\sigma$. This value determine the saturation intensity of the amplifier medium and is the reason why a Nd:YAG amplifier cannot be used. Because of their low cross sections, $\mathrm{Cr}^{+}$active materials such as ruby, alexandrite, and other chromium garnet lasers should easily meet the second threshold condition.

Equation (9) and expression (23) are both necessary and sufficient conditions for obtaining the $Q$ switching of the cavity in a self-focusing $Q$-switching system. The pumping rate $P$ is a negligible quantity in the majority of practical cases, compared with the two terms of Eq. (9) and expression (23). Hence it is possible to obtain a simpler expression by neglecting this quantity, and we obtain a necessary but insufficient condition as

$$
\frac{\sigma T_{\text {cav }} A\left(g_{0}-1\right)^{1 / 2}}{\beta h \nu}-\frac{2(A-L-\Gamma)}{\left(g_{0}+1\right)^{1 / 2}}<0 .
$$

It must be pointed out that the pumping rate $P$ must be considered a crucial parameter in $k_{\text {sat }}$ evaluation and therefore in the amplification and loss coefficients $A$ and $L$.

We have presented a master equation that defines the $Q$ switching condition in a self-focusing $Q$-switching system. This equation includes the principal laser parameters that can be evaluated $a$ priori with a complete set of equations. By using the condition derived, it was shown that with the nonlinear material used in the previous work (phenyl salicylate), the second threshold condition cannot be achieved for a Nd:YAG laser. For a Nd:glass laser the best configuration is with a positive-branch resonator. The second threshold condition cannot be achieved with a negative-branch resonator. The equation presented can easily be applied to other nonlinear materials or amplifier media in order to determine if the $Q$-switching operation can be obtained.

\section{ACKNOWLEDGMENT}

This research was partially supported by a grant from the Organization of American States.

M. C. Marconi is a Conicet Fellow. O. E. Martinez is a member of the Carrera del Investigador del Consejo Nacional de Investigaciones Cientificas y Tecnicas de la Republica Argentina.

\section{REFERENCES}

1. M. C. Marconi, O. E. Martinez, and F. P. Diodati, Opt. Lett. 10, 402 (1985).

2. A. G. Fox and T. Li, Bell Sys. Tech. J. 40, 1 (1961).

3. A. G. Fox and T. Li, Proc. IEEE 51, 80 (1963).

4. R. J. Collier, C. B. Burckhardt, and L. H. Lin, Optical Holography (Academic, New York, 1971).

5. H. Kogelnik and T Li, Appl. Opt. 10, 1550 (1966).

6. G. H. C. New and T. B. O'Hare, Phys. Lett. 68A, 1 (1978).

7. G. H. C. New, Proc. IEEE 67, 380 (1979).

8. A. E. Siegman, Proc. IEEE 53, 277 (1965).

9. A. E. Siegman, An Introduction to Lasers and Masers (McGraw-Hill, New York, 1971).

10. L. W. Casperson, IEEE J. Quantum Electron. QE-10, 9 (1974).

11. A. E. Siegman, IEEE J. Quantum Electron. QE-12, 1 (1976).

12. W. Koechner, Solid State Laser Engineering (Springer-Verlag, New York, 1976), p 589

13. M. Abramowitz and I. Stegun, Handbook of Mathematical Functions (Dover, New York, 1970).

14. The main characteristics of the phenil salycilate was obtained from P. P. Ho and R. R. Alfano, J. Chem. Phys. 67, 3 (1977); see also Phys. Rev. A 20, 5 (1979). 